\title{
Änderungen der KLV, AL und MiGeL
}

\author{
Susanne Christen ${ }^{a}$, Thomas Kessler ${ }^{b}$ \\ ${ }^{a}$ Dr. med., FMH, stv. Abteilungsleiterin, Abteilung Ambulante Versorgung und Tarife; ${ }^{b}$ FMH, Experte, Abteilung Ambulante Versorgung und Tarife
}

In diesem Artikel werden die wichtigsten Änderungen und Anpassungen der Krankenleistungsverordnung KLV, der Analysenliste AL und der Mittel- und Gegenständeliste MiGeL per 1.3.2019, 1.4.2019 und 1.7.2019 aufgezeigt.

Krankenleistungsverordnung KLV: Für Art. 12a Bst. a, b, d und g KLV gilt neu der Impfplan 2019. Zusätzlich gilt für die Meningokokken-Impfung: Gemäss Impfplan 2018 und den Empfehlungen des BAG und der EKIF vom 22. Oktober 2018 (BAG-Bulletin Nr. 43/18). Für Art. 12d Bst. a KLV HIV-Test gilt neu die Richtlinie «Der HIV-Test auf Initiative des Arztes / der Ärztin bei bestimmten Krankheitsbildern (HIV-Indikatorerkrankungen)» des BAG vom 18. Mai 2015. Art. 12e Bst. d KLV Früherkennung des Kolonkarzinoms wird mit dem Früherkennungsprogramm des Kantons Wallis ergänzt. Nach Art. 13 Bst. b $^{\text {ter }}$ KLV Nicht-invasiver pränataler Test (NIPT) ist neu der Expertenbrief Nr. 52 vom 14. März 2018 der Schweizerischen Gesellschaft für Gynäkologie und Geburtshilfe (SGGG) zu berücksichtigen. Zusätzlich gilt: Bei Zwillingsschwangerschaften sind NIPT mittels Microarray oder Single Nucleotide Polymorphism (SNP) von der Kostenübernahme durch die Versicherung ausgeschlossen.

Krankenleistungsverordnung KLV Anhang 1: Neu ist gemäss Punkt 1.4 Urologie und Proktologie Anhang 1 KLV die perkutane Elektroneuromodulation des Tibialnervs ab dem 1.3.2019 eine Pflichtleistung zur Behandlung der idiopathischen hyperaktiven Blase oder der Stuhlinkontinenz. Dies nach Ausschöpfen konservativer Therapieoptionen. Für Punkt 2.1 Innere Medizin Allgemein Anhang 1 KLV gilt für die hämatopoïetische Stammzell-Transplantation ab dem 1.3.2019 die 7. Ausgabe vom März 2018 der «FACT-JACIE International Standards for hematopoietic Cellular Therapy Product Collection, Processing and Administration». Für Punkt 2.3 Neurologie inkl. Schmerztherapie und Anästhesie Anhang $1 \mathrm{KLV}$ gilt für die fokussierte Ultraschalltherapie im Pallidum, Thalamus und Subthalamus bis zum 30.6.2020 zusätzlich neu: Zur Behandlung bei etablierter Diagnose einer idiopathischen parkinsonschen Krankheit, Progredienz der Krankheitssymptome über mindestens 2 Jahre. Ungenügende Symptomkontrolle durch Dopamin-Behandlung (Off-Phänomen, On-/OffFluktuationen, On-Dyskinesien). Gemäss Punkt 5 Dermatologie Anhang $1 \mathrm{KLV}$ ist neu die Anwendung von Hautäquivalenten für die Behandlung von chronischen Wunden unter bestimmten Voraussetzungen ab dem 1.3.2019 eine Pflichtleistung. Gemäss Punkt 9 Radiologie Anhang 1 KLV Positron-Emissions-Tomographie (PET, PET/CT) gibt es diverse Änderungen. So kann in der Kardiologie bei Verdacht auf eine Infektion von kardiologischen Implantaten entsprechende Bildgebung ab dem 1.3.2019 gemacht werden. Neu ergänzt kann in der Inneren Medizin / Infektiologie entsprechende Bildgebung z.B. bei Fieber unbekannter Ursache, nach inkonklusiver internistischer und infektiologischer Abklärung und Bildgebung sowie bei Verdacht auf eine Infektion von vaskulären Grafts oder bei alveolärer Echinokokkose im Hinblick auf ein mögliches Absetzen der medikamentösen Therapie erbracht werden. In Evaluation ist die Bildgebung zur präoperativen Lokalisation Nebenschilddrüsen-Adenomen bei primärem Hyperparathyreoidismus nach negativer oder inkonklusiver konventioneller Bildgebung (Sestamibi-Szintigraphie bzw. -SPECT/CT).

Alle Änderungen der KLV inklusive Anhang 1 im Detail finden Sie unter diesem Link des Bundesamtes für Gesundheit BAG: www.bag.admin.ch $\rightarrow$ Gesetze \& Bewilligungen $\rightarrow$ Gesetzgebung $\rightarrow$ Gesetzgebung Versicherungen $\rightarrow$ Gesetzgebung Krankenversicherung $\rightarrow$ Bundesgesetz über die Krankenversicherung $\rightarrow$ Änderungen in der Krankenpflege-Leistungsverordnung (KLV)

\section{Änderungen der Analysenliste AL per 1.3.2019}

Für das Praxislabor wurde der Zuschlag 4707.10 für jede Analyse, die das Suffix C aufweist, für Analysen der Ziffer 5.1.2.2.2 «Ergänzende Analysen» und Analysen 
der Ziffer 5.1.3 «Analysen für Ärzte oder Ärztinnen mit bestimmten Weiterbildungstiteln» beibehalten, jedoch für Analysen der Ziffer 5.1.4 «Analysen für Ärzte oder Ärztinnen zur Durchführung anlässlich eines Hausbesuchs» gestrichen.

Die Analyse 1735.00 "Troponin, T oder I, Schnelltest» wurde schon per 1.9.2018 gestrichen, damals aber für die 5.1.4. "Analysen für Ärzte oder Ärztinnen zur Durchführung im Rahmen eines Hausbesuchs» beibehalten. Neu wird nun auch für den Hausbesuch diese Analyseposition 1750.00 gestrichen, dafür kann aber nun die Analyseposition 1734.01 «Troponin, T oder I, qn, mittels Immunoassay" verwendet werden. Dabei sind folgende Limitationen zu beachten: «nicht mittels Schnelltests (qualitative Bestimmung), nicht kumulierbar mit Position 1249.01 'Creatin-Kinase (CK), total', die Kostenübernahme ist befristet bis am 30. Juni 2021».

Alle Änderungen der Analysenliste im Detail finden Sie unter diesem Link des Bundesamtes für Gesundheit BAG: www.bag.admin.ch/al

\section{Änderungen der Mittel- und Gegenstände- liste MiGeL per 1.4.2019}

Unter Punkt 4.6 wird "pro Jahr" und "pro rata" genauer definiert und erklärt:

«Eine Therapie, bzw. der Bezug von entsprechenden Produkten beginnt häufig nicht am 1. Januar eines Jahres. Die Vergütung bei einem HVB 'pro Jahr (pro rata)' bezieht sich immer auf den Anteil des Kalenderjahres, in dem die Therapie effektiv genutzt wurde.

Ein Beispiel: Es erfolgt ein erstmaliger Bezug von regelmässig zu nutzendem Verbrauchsmaterial mit einem HVB von CHF 400.- pro Jahr pro rata ab dem 1. Oktober. $\mathrm{Zu}$ vergüten ist im Jahr des Erstbezugs der entsprechende Anteil des HVB, der für dieses Kalenderjahr bleibt (3 Monate), also pro rata CHF 100.-. Im folgenden Jahr wird die Therapie dauerhaft fortgesetzt und es kann maximal CHF 400.- für das ganze Kalenderjahr vergütet werden. Als Rundungsregel gilt dabei, dass üblicherweise die Vergütung auf ganze Stückzahlen aufzurunden ist, z.B. wenn ein Produkt alle 2 Wochen zu erneuern ist.

HVB 'pro Jahr' werden meist bei Geräten oder Hilfsmitteln verwendet, die nicht mehrfach pro Jahr gekauft werden, oder die unregelmässig anzuwenden sind. Sie verstehen sich als maximal mögliche Vergütung in den 365 Tagen ab dem ersten Bezugsdatum. Das heisst nicht pro Kalenderjahr, sondern pro Jahr der laufenden Therapie.

Ein Beispiel: Brillengläser/Kontaktlinsen werden bis zum vollendeten 18 . Altersjahr maximal $1 \mathrm{Mal}$ pro Jahr mit einem HVB vergütet. Erfolgt der erste Bezug am 31. Oktober 2015, kann frühestens für einen Bezug im November 2016 erneut eine Vergütung erfolgen.

Als Ausnahme kann auch mehrfach pro Jahr zu kaufendes, aber unregelmässig zu nutzendes Material einen HVB pro Jahr erhalten. Ein Beispiel sind BlutzuckerMessstreifen bei nicht insulinpflichtigen Diabetikern. Hier ist die einmal tägliche (einer pro rata-Vergütung entsprechende) Anwendung nur in wenigen Ausnahmefällen medizinisch sinnvoll. Hingegen sollen kurzzeitige Perioden mit intensiveren Messungen, z.B. für Therapieumstellungen, ermöglicht werden. Gleichzeitig wird so berücksichtigt, dass auch bei einem Beginn mit Blutzuckermessungen gegen Ende Jahr zunächst eine Kleinpackung Messstreifen vergütet werden kann.» Zudem gibt es zum Teil sehr umfassende Änderungen in folgenden Kapiteln:

15. Inkontinenzhilfen

17. Kompressionstherapie-Mittel (inkl. Stützstrümpfen)

29. Stomaartikel

35. Verbandmaterial

Alle Änderungen der Mittel- und Gegenständeliste im Detail finden Sie unter diesem Link des Bundesamtes für Gesundheit BAG: www.bag.admin.ch/migel 